\title{
Pobreza y procedencia indígena como factores de riesgo de problemas nutricionales de los niños que ingresan a la escuela
}

\author{
Patricia Bustos, MC,(1) Sergio Muñoz, PhD Bioestadística, ${ }^{(2)}$ Claudio Vargas, MC, (1) \\ Hugo Amigo, Dr en SP.(1)
}

\begin{abstract}
Bustos P, Muñoz S, Vargas C, Amigo H. Pobreza y procedencia indígena como factores de riesgo de problemas nutricionales de los niños que ingresan a la escuela. Salud Publica Mex 2009;5 I:187-193.
\end{abstract}

\section{Resumen}

Objetivo. Determinar el efecto de ser indígena y de la pobreza, en los problemas nutricionales de escolares chilenos. Material y métodos. Se usaron bases de datos de niños que ingresaron a la escuela (1997-2004) que tenían información antropométrica, socioeconómica y origen étnico.Se construyeron modelos de regresión logística para talla baja y obesidad controlando el efecto de la pobreza y la etnia por sexo, edad y año de análisis. Resultados. Se estudió a I 580103 niños: indígenas $(7.4 \%)$, con talla baja $(2.9 \%)$ y obesidad (16\%). Al estratificar por etnia, los más pobres tuvieron mayor riesgo de talla baja: en indígenas RM: 2.30 (IC95\%: 2.27-2.33) y no indígenas $R M: 2.29$ (IC95\%: 2.28-2.30). A la inversa, los escolares más pobres tuvieron menos riesgo de ser obesos (RM: 0.63; IC95\%: 0.62-0.64). Ser indígena proporcionó $6 \%$ más posibilidad de presentar obesidad, comparado con no ser indígena (RM: I.06; IC95\%: I.05-I.08). Conclusión. En niños chilenos, la pobreza es factor de riesgo de talla baja pero protector de obesidad independiente de la etnia. El mayor riesgo de obesidad en los escolares indígenas, si bien es pequeño, debe ser una voz de alerta para prevenir en ellos el aumento de las cifras.

Palabras clave: pobreza; indígenas; baja talla; obesidad; Chile
Bustos P, Muñoz S, Vargas C, Amigo H.

Poverty and indigenous origin as risk factors

of nutritional problems among children who enroll in school.

Salud Publica Mex 2009;5 I:187-193.

\begin{abstract}
Objective. To estimate the effect of indigenous ancestry and poverty on nutritional outcomes in Chilean schoolchildren. Material and Methods. We used the national database of children entering to the public educational system in 1997-2004. This includes anthropometric assessment, socioeconomic status and parental surnames, used to derive the ethnic origin. Logistic regression models related poverty and ethnicity on stunting and obesity were done, controlling for sex, age and calendar year. Results. Data convey I 580103 children being $7.4 \%$ indigenous; $2.9 \%$ had stunting and around $16.0 \%$ were obese. Stratifying by poverty, it was shown that the poorest had higher risk of stunting both in indigenous $(O R=2.30 ; C l 95 \%=2.27-2.33)$ and non indigenous $(O R=$ $2.29 ; \mathrm{Cl} 95 \%=2.28-2.30)$. Conversely, poverty was a "protective factor" for obesity $(\mathrm{OR}=0.63 ; \mathrm{Cl} 95 \%=0.62-0.64)$. Indigenous origin showed a significant OR slightly over the null. Conclusions. In Chilean children, poverty is a risk factor for stunting but still protects from obesity, independent of indigenous origin.
\end{abstract}

Key words: poverty; indigenous; stunting; obesity; Chile

Este trabajo recibió financiamiento del Fondo Nacional de Investigación en Salud de Chile (FONIS, proyecto SA05i20077).

(I) Departamento Nutrición, Facultad de Medicina, Universidad de Chile. Santiago, Chile.

(2) Departamento Salud Pública, Facultad de Medicina, Universidad de La Frontera. Temuco, Chile.

Fecha de recibido: 17 de junio de 2008 - Fecha de aceptado: 20 de febrero de 2009 Solicitud de sobretiros: Patricia Bustos. Depto. Nutrición, Facultad de Medicina, Universidad de Chile. Independencia 1027, Santiago, Chile. Correo electrónico:pbustos@med.uchile.cl 
L a identificación y vigilancia de los problemas nutricionales colectivos son una preocupación constante de todos los gobiernos y, en general, se han observado mejorías notorias para los problemas por déficit, aunque éstos todavía persisten, sobre todo en los lugares más pobres o donde vive la población indígena. Con respecto al exceso de peso, la situación es diferente al observarse un aumento progresivo; en consecuencia, coexisten los problemas por déficit y exceso de peso en la mayor parte de los países de América Latina.

La baja talla es un problema nutricional que aún afecta a los niños del continente. En algunos países de América Central, la prevalencia es elevada; en censos de talla realizados en escolares que ingresan al primer grado básico en El Salvador (2000), Guatemala (2001) y Honduras (1997) se han reportado cifras de 3.1, 14.5 y $15.2 \%$, respectivamente, de niños ubicados bajo -3 desviaciones estándar. ${ }^{1}$ Este problema se concentra en el nivel socioeconómico bajo y los grupos indígenas. En la encuesta nacional de nutrición que se viene realizando periódicamente en México se indica una prevalencia de talla baja de $44.3 \%$ en niños indígenas, comparada con $14.5 \%$ en los no indígenas. ${ }^{2,3}$ La explicación que se ha dado a las mayores cifras de talla baja en población indígena es una combinación de factores étnicos (genéticos) y ambientales al concentrarse en ellos, por generaciones, la pobreza. ${ }^{4}$

El exceso de peso en la población indígena se ha estudiado menos dado que es un problema nutricional de aparición más reciente. Los datos disponibles en países como Estados Unidos de América y Canadá muestran que en el nivel socioeconómico bajo el exceso de peso de los niños indígenas es más frecuente que el observado en niños no indígenas. ${ }^{5-7}$ La explicación aducida es el cambio de las costumbres alimentarias (aumento del consumo y cambio en la proporción de macronutrientes) y el mayor sedentarismo que han tenido estas poblaciones en décadas recientes. ${ }^{8}$

En Chile, así como en otros países de América Latina, las estadísticas nacionales se han desagregado en escasa medida por condición social, y mucho menos por procedencia indígena, aunque existen algunos antecedentes que permiten hacerlo. Presentar información desagregada por estas características es una necesidad, toda vez que en estos grupos poblacionales los problemas nutricionales se presentan en mayor magnitud y porque los gobiernos han declarado sus intenciones de reducir la inequidad social al permitir que los grupos poblacionales más desprotegidos sean los principales beneficiarios de programas de apoyo social o nutricional.

El objetivo de este trabajo fue determinar el efecto de la etnia y la vulnerabilidad social (como una indica- ción del nivel socioeconómico) en la talla baja y obesidad de niños chilenos de primer año básico. Este trabajo se centra en la población que ingresa a la enseñanza básica porque el registro de datos antropométricos se efectúa con un procedimiento normado, en forma ininterrumpida, desde hace más de 20 años, y porque a esta edad el estado nutricional de los niños refleja su historial de salud y nutrición. ${ }^{9}$

\section{Material y métodos}

La situación nutricional se evaluó mediante las mediciones de todos los niños que ingresaron a primer año básico en escuelas públicas o particulares subvencionadas por el Estado, entre los años de 1997 y 2004, y que proporcionó el Ministerio de Educación de Chile. Las mediciones se llevaron a cabo al comienzo de cada año escolar por profesores, que han sido capacitados y disponen de un instructivo para la realización estandarizada de ellas. ${ }^{10}$ Entre las consideraciones que se deben aplicar -según el instructivo- figuran las siguientes: calibrar la balanza antes de cada medición y efectuar la medición en ropa interior (y sin zapatos); la estatura se debe medir con ropa mínima y sin zapatos, apoyando talones, glúteos, espalda y cabeza al plano vertical. La cabeza debe erguirse de tal manera que ojos y orejas se hallen a la misma altura. Los datos que se presentan corresponden a alrededor de $70 \%$ del total de los niños chilenos que ingresan a la enseñanza básica (con una cobertura de las mediciones que alcanza 88\%); el porcentaje restante asiste a escuelas particulares y no se incluyó en la información analizada; estos últimos escolares pertenecen a un nivel socioeconómico más privilegiado, en el cual la proporción de niños de procedencia indígena es muy baja.

Para el estudio de la estatura se expresó la talla en puntajes $\mathrm{z}$, de acuerdo con la edad y el sexo; se consideró talla baja a los niños ubicados $\leq-2$ puntajes z. El exceso de peso se cuantificó con base en el índice de masa corporal (IMC) y se consideraron obesos los individuos que estaban $\geq 2$ puntajes z. Para el cálculo de ambos índices se utilizaron como referencia las tablas del CDC/ NCHS. ${ }^{11}$

En este estudio se incluyó a los escolares que ingresaban por primera vez a la escuela; por lo tanto, los que tenían una segunda medición (repitentes) se excluyeron. Los datos se analizaron tras eliminar los casos con mediciones faltantes; en el caso de la estatura, también se excluyó a los niños con datos alejados de un rango considerado posible para la situación actual del país $( \pm$ 4 puntajes z) cuyo porcentaje no superó $1 \%$ en todos los años estudiados. En el caso del IMC no se excluyeron los valores, incluso si eran muy altos. 
La procedencia étnica se analizó según fuera el número de apellidos mapuche y los datos se dividieron en dos grupos: con y sin esta procedencia. Para su identificación se construyó un catastro de apellidos con datos provenientes de diversas fuentes: libros de historia del pueblo mapuche, registros eclesiásticos, beca indígena que otorga el Ministerio de Educación y postulantes a beneficios o inscritos en la Corporación de Desarrollo Indígena (organismo público destinado a preservar la cultura y el patrimonio de las etnias indígenas del país). Posteriormente, especialistas en la lengua mapuche los revisaron y se obtuvo al final un listado con 7306 apellidos de este origen.

La vulnerabilidad social se calculó al considerar tres variables que se registran para cada niño en la ficha de evaluación que completan los profesores: a) puntaje en una encuesta socioeconómica (ficha CAS) ${ }_{1}^{12}$ b) pertenecer (o no) al programa Chile Solidario y c) ubicación según previsión en salud. Los datos de la ficha CAS se obtuvieron de una entrevista domiciliaria que aplican todas las municipalidades del país para conocer la situación socioeconómica de la familia, requisito imprescindible para la postulación a los subsidios que entrega el Estado a las familias en situación de pobreza. Se consideran 13 variables distribuidas en cuatro factores (vivienda, educación, ocupación, ingreso / patrimonio). Chile Solidario es un sistema público de protección social dirigido a las familias en situación de extrema pobreza, cuyo objetivo es promover su incorporación a las redes sociales y su acceso a mejores condiciones de vida (apoyo psicosocial, acceso al subsidio familiar y pensiones asistenciales). En relación con la atención de salud, las posibilidades eran: pertenecer al sistema público de atención o atenderse a través a un sistema privado.

Con esta información se construyeron tres categorías de vulnerabilidad: niños cuyo puntaje de la ficha CAS correspondía al tercio inferior de frecuencias, pertenecer al programa Chile Solidario y tener gratuidad en la atención de salud (denominada "alta" vulnerabilidad y usada como sinónimo de mayor pobreza). A la inversa, el grupo con menor vulnerabilidad incluyó a niños cuyo puntaje en la ficha CAS estuvo en el tercio de mejor puntuación, no pertenecían a Chile Solidario y su atención de salud era a través del sistema privado (este grupo constituyó la "baja vulnerabilidad" que corresponde a la clase media del país). Se conformó también un grupo de vulnerabilidad media cuyo puntaje CAS estaba en un nivel intermedio; su atención de salud, si bien se proporcionaba a través del sistema público, se hallaba en los tramos mejores y podían o no pertenecer al programa Chile Solidario.

Se construyó una gran base de datos que agrupó a ocho bases distintas (una por año, de 1997 a 2004). Se hicieron estimaciones de prevalencia por etnia y sexo y se construyeron modelos de regresión logística en los que la variable de desenlace fue una variable indicadora de talla baja u obesidad controlando el efecto de pobreza y etnia por sexo, edad y año de análisis. La población de menor vulnerabilidad fue el grupo de referencia para el análisis de la condición social.

Este proyecto recibió aprobación del Comité de Ética de la Facultad de Medicina de la Universidad de Chile y contó con la autorización de la Junta Nacional de Auxilio Escolar y Becas (JUNAEB), organismo autónomo del Ministerio de Educación de Chile, para su realización. Como el estudio consistió en el análisis de las bases de datos nacionales, no se obtuvo el consentimiento informado de los participantes.

\section{Resultados}

El análisis incluyó datos de 1580103 escolares, de los cuales $7.43 \%$ tenía procedencia mapuche. La edad promedio de los niños del estudio fue de seis años cuatro meses, levemente mayor en los mapuche, con similar proporción de hombres y mujeres. Desde el punto de vista de la vulnerabilidad social, algo más de $10 \%$ fue calificado como de alta vulnerabilidad o pobreza y esta proporción fue significativamente mayor en la población de procedencia indígena en la cual 1 de cada 4 escolares se ubicaba en la categoría de pobreza, al contrario de los de baja vulnerabilidad, en quienes la mayoría no pertenece al grupo indígena $(p<0.001)$. Los promedios de peso fueron un poco mayores en los individuos no indígenas, al igual que la talla en la que la diferencia en favor de ellos es casi de $1 \mathrm{~cm}$; en cambio, el IMC fue mayor en los indígenas.

La talla baja comprometió a una proporción algo inferior a 3\% de los escolares analizados, apenas mayor de lo esperado (según referencia, 2.3\% de la población debería estar ubicada $<-2$ puntajes $\mathrm{z}$ ), aunque esta proporción fue mayor en niños mapuche, al superar $4 \%(p<0.001)$; por el contrario, las cifras de obesidad fueron elevadas, cercanas a 16\% (se esperaría 2.3\% sobre 2 puntajes z) y con proporciones similares en los dos grupos étnicos analizados (cuadro I).

Se observó un aumento significativo de las prevalencias de talla baja a medida que la vulnerabilidad social era mayor (mayor pobreza), tanto en niños mapuches como en no mapuches. Las cifras del déficit de talla en la elevada vulnerabilidad duplican las encontradas en el grupo menos pobre en ambos grupos étnicos. Lo contrario se observó con las cifras de obesidad, en las que se advirtió que a medida que la vulnerabilidad social aumentaba, las prevalencias disminuían en ambos grupos étnicos (cuadro II). 
$\mathrm{Al}$ analizar en forma conjunta el efecto de la etnia y la vulnerabilidad social, controlando por edad, sexo y año de medición, se identificó que hay un efecto dosis-respuesta: a medida que aumenta la pobreza, también lo hace el riesgo relacionado con la talla baja. Ser mapuche tuvo un valor menor, aunque superior a la unidad y significativo (cuadro III). Dado que se identificó una interacción entre el grupo étnico y la vulnerabilidad social, se realizaron análisis estratificados por etnia y se observó que la pobreza tuvo efectos muy similares con riesgos de $2.30($ IC95\% $=2.27-2.33)$ para los indígenas y $2.29($ IC95\% $=2.28-2.30)$ para los no indígenas si éstos pertenecían a la categoría de los más pobres.

Cuadro I

Características generales de los escolares de primer año básico SEgúN SU PROCEDENCIA INDíGENA O NO INDÍGENA. CHILE, 1997-2004

\begin{tabular}{|c|c|c|c|c|}
\hline Variable & 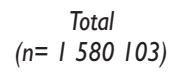 & $\begin{array}{l}\text { Mapuche } \\
\text { (II7 II3) }\end{array}$ & $\begin{array}{l}\text { No mapuche } \\
\text { (I } 462 \text { 990) }\end{array}$ & $p$ \\
\hline Edad en meses: promedio $\pm \mathrm{DE}$ & $76.3 \pm 4.35$ & $76.7 \pm 4.81$ & $76.2 \pm 4.31$ & 0.000 \\
\hline$\%$ varones & 50.9 & 51.2 & 50.9 & 0.077 \\
\hline \multicolumn{5}{|l|}{ Vulnerabilidad (\%) } \\
\hline Baja $(n=1015983)$ & 64.3 & 49.3 & 65.5 & \\
\hline Media $(n=364745)$ & 23.1 & 24.2 & 23 & 0.001 \\
\hline Alta $(n=199375)$ & 12.6 & 26.5 & 11.5 & \\
\hline Peso $(\mathrm{kg})$ promedio $\pm \mathrm{DE}$ & $23.06 \pm 4.33$ & $22.89 \pm 4.24$ & $23.07 \pm 4.34$ & 0.001 \\
\hline Estatura $(\mathrm{cm})$ promedio $\pm \mathrm{DE}$ & $117.4 \pm 5.82$ & $116.7 \pm 5.84$ & $117.5 \pm 5.8 \mathrm{I}$ & 0.001 \\
\hline IMC (puntajes z) promedio $\pm \mathrm{DE}$ & $0.76 \pm 1.44$ & $0.80 \pm 1.42$ & $0.75 \pm 1.44$ & 0.001 \\
\hline
\end{tabular}

Cuadro II

Proporción de ESCOLARES de PRIMER AÑo bÁSICO CON TALLA bajA Y OBESIDAD SEGÚN LA VULNERABILIDAD SOCIAL Y EL GRUPO ÉTNICO. CHILe, I 997-2004

\begin{tabular}{|c|c|c|c|c|c|}
\hline \multirow[b]{2}{*}{ Problema nutricional } & \multirow[b]{2}{*}{ Total } & \multicolumn{3}{|c|}{ Vulnerabilidad } & \multirow[b]{2}{*}{$p$} \\
\hline & & Baja & Mediana & Alta & \\
\hline \multicolumn{6}{|l|}{ Talla baja } \\
\hline Mapuche (\%) & 4.2 & 3.1 & 4.0 & 6.6 & $0.001 *$ \\
\hline No mapuche (\%) & 2.8 & 2.3 & 3.4 & 4.8 & $0.001 *$ \\
\hline$p^{\ddagger}$ & 0.001 & & & & \\
\hline \multicolumn{6}{|l|}{ Obesidad } \\
\hline Mapuche (\%) & 15.8 & 17.9 & 15.3 & 12.3 & $0.001 *$ \\
\hline No mapuche (\%) & 15.9 & 17.1 & 14.6 & 11.7 & $0.001 *$ \\
\hline$p^{\ddagger}$ & 0.143 & & & & \\
\hline
\end{tabular}




\section{Cuadro III}

Procedencia étNica Y VUlNerabilidad social como factores de Riesgo de talla baja en escolares ChILENOS de PRIMER AÑo básıco. ChILe, I997-2004*

\begin{tabular}{|c|c|c|c|c|c|}
\hline \multirow[b]{2}{*}{ Variable } & & \multirow[b]{2}{*}{$R M$} & \multicolumn{2}{|c|}{ IC95\% } & \multirow[b]{2}{*}{ Significancia } \\
\hline & & & Inferior & Superior & \\
\hline Etnia & No mapuche & 1.00 & & & \\
\hline & Mapuche & 1.26 & 1.22 & 1.30 & 0.001 \\
\hline \multirow[t]{3}{*}{ Vulnerabilidad social } & Baja & 1.00 & & & \\
\hline & Media & 1.43 & 1.40 & 1.47 & 0.001 \\
\hline & Alta & 2.03 & 1.98 & 2.08 & 0.001 \\
\hline
\end{tabular}

* Estimaciones basadas en un modelo de regresión logística múltiple ajustado por sexo, edad y año de análisis

Cuadro IV

Procedencia étNica Y VUlnerabilidad social como factores de RIESgo de obesidad en escolares Chilenos de Primer año básico. Chile, 1997-2004*

\begin{tabular}{|c|c|c|c|c|c|}
\hline \multirow[b]{2}{*}{ Variable } & & \multirow[b]{2}{*}{$R M$} & \multicolumn{2}{|c|}{ IC95\% } & \multirow[b]{2}{*}{ Significancia } \\
\hline & & & Inferior & Superior & \\
\hline \multirow[t]{2}{*}{ Etnia } & No mapuche & 1.00 & & & \\
\hline & Mapuche & 1.06 & 1.05 & 1.08 & 0.001 \\
\hline \multirow[t]{3}{*}{ Vulnerabilidad social } & Baja & 1.00 & & & \\
\hline & Media & 0.824 & 0.815 & 0.833 & 0.001 \\
\hline & Alta & 0.633 & 0.623 & 0.642 & 0.001 \\
\hline
\end{tabular}

* Estimaciones basadas en un modelo de regresión logística múltiple ajustado por sexo, edad y año de análisis

En el caso de la obesidad, la situación fue diferente: pertenecer a los grupos de mayor pobreza constituyó un factor protector ( $R M: 0.633)$, al igual que el grupo de pobreza intermedia (RM: 0.824), mientras que tener procedencia mapuche presenta una RM levemente superior a la unidad también significativa (cuadro IV). No hubo interacción entre vulnerabilidad social y grupo étnico.

\section{Discusión}

En este estudio, realizado en más de 1500000 escolares de primero básico, se desprenden dos tipos de conclusiones. En relación con la talla baja, el principal factor relacionado es la pobreza, al margen de pertenecer a la etnia indígena. En cuanto a la obesidad, la pobreza constituye un factor protector al encontrarse que los escolares que están en mejores condiciones tienen ma- yor prevalencia de obesidad, sin relación notoria con la procedencia indígena.

El efecto de las desigualdades sociales y la etnia en la determinación de la talla baja se describió previamente en un estudio que analizó la situación de cuatro países andinos; en esos protocolos se identificaron cifras elevadas de déficit de talla, sobre todo en la población indígena (en Bolivia, Perú y Ecuador era el doble respecto de la población no indígena) y un gradiente socioeconómico en el cual la talla baja era mayor en los más pobres en comparación con la observada en los deciles superiores. En Colombia, si bien se reconoció una situación parecida, el gradiente fue menor. ${ }^{13}$ Un análisis más detallado efectuado en Ecuador demostró que el factor más importante vinculado con la talla baja era la desigualdad económica a nivel provincial, incluso con control de etnia indígena y vivir en altura. ${ }^{14}$ 
El efecto de la pobreza y la etnia obtenido en este estudio es consistente con lo descrito en los países andinos mencionados, aunque la prevalencia del déficit de talla en escolares chilenos es menor y refuerza los hallazgos de un trabajo previo llevado a cabo en el país en una muestra de escolares de primer año básico; en esta investigación se comprobó que el retardo de crecimiento en estatura se relacionaba con la pobreza tanto en niños de procedencia mapuche como en los no mapuche, no tanto con el origen étnico. ${ }^{15}$ En el análisis de las curvas de crecimiento de esos mismos niños desde el nacimiento se observó que el menor incremento en talla era de inicio posnatal temprano, en particular en los de origen indígena de zonas de muy alta pobreza. ${ }^{16}$

Las explicaciones de los nexos encontrados probablemente se encuentran en la secuencia ya descrita de pobreza, alimentación inadecuada, déficit de peso crónico junto con enfermedades infecciosas durante los primeros años de vida, y factores que comprometen el crecimiento de estatura y determinan la talla baja, manifestados de forma más marcada en la edad escolar. ${ }^{17}$ $\mathrm{Al}$ ser la talla baja -a escala poblacional- consecuencia de una desnutrición en etapas tempranas de la vida, la información de estos estudios refuerza la importancia de focalizar los programas de salud, alimentación y prevención de la desnutrición en la población indígena, en los municipios más pobres y desde muy temprano de la vida.

Al contrario de lo esperado, la prevalencia de obesidad es mayor en los escolares menos pobres, aunque sin diferenciación por etnia. En mujeres adultas chilenas, el gradiente de obesidad por nivel socioeconómico es bien marcado y mayor en las de menor nivel socioeconómico. ${ }^{18}$ La característica encontrada en este trabajo, en el cual la obesidad no se concentra en los más pobres, ya se describió en poblaciones que han superado un nivel de pobreza marcado en el que emergen grupos que tienen un mayor poder adquisitivo que les permitiría la compra de alimentos con alto contenido energético y tal vez menor actividad física. ${ }^{19}$ Los de mejor nivel socioeconómico del país -que están excluidos de este análisis ya que probablemente asisten a colegios privados- quizá no tienen cifras de obesidad tan altas como las obtenidas en este trabajo y sería interesante mostrarlas.

Las altas cifras de obesidad encontradas en niños indígenas tal vez reflejen los cambios que han experimentado estas comunidades en el país, con abandono de los hábitos alimentarios autóctonos que los caracterizaban y un creciente sedentarismo, al desplazarse en autobuses escolares aun en el área rural, disminución del trabajo manual y escasa actividad física y recreativa en las escuelas. En un estudio llevado a cabo en escolares indígenas y no indígenas de distinta vulnerabilidad social se pudo constatar que no hubo diferencias significativas en la ingesta calórica entre etnias, aunque se observó que a medida que decrecía la pobreza se incrementaba la ingestión energética y de macronutrientes. ${ }^{20}$

El mayor riesgo de obesidad en los escolares indígenas, si bien es pequeño, debe ser una voz de alerta para prevenir en ellos el aumento de las cifras; este aspecto, como se señaló, se ha identificado en años recientes en niños indígenas provenientes de países con mayor desarrollo, como Estados Unidos de América y Canadá. $5-8$

Ya se han descrito con anterioridad ${ }^{21}$ el riesgo de talla baja disminuido y el de obesidad aumentado, lo que refleja la evolución nutricional del país. Ésta tuvo una rápida transición epidemiológica con disminución notoria de la desnutrición global y de micronutrientes, pero con aumento del exceso de peso y las enfermedades crónicas no trasmisibles relacionadas con él.

Entre las limitaciones de este estudio están el trabajo con datos secundarios y las mediciones antropométricas realizadas por profesores. En estudios previos efectuados en el país se ha comprobado que la concordancia entre las mediciones realizadas por los profesores y un equipo técnico especializado es baja; empero, aunque hay discrepancias entre las mediciones, los altos niveles de peso excesivo o bajo se mantienen y reflejan problemas con exactitud y sin sesgo.22 También es una limitante no contar con un indicador más directo de la condición socioeconómica de los niños, aunque en esta oportunidad se realizó un esfuerzo para aglutinar toda la información socioeconómica disponible para clasificar la población estudiada.

Trabajar sólo con los escolares mapuche limita los resultados a esta etnia, pero cabe señalar que es el grupo mayoritario de indígenas de Chile. También puede ser cuestionable la identificación de la etnia a través de los apellidos, pero ésta parece una buena forma de hacerlo en estudios poblacionales que abarcan a un gran número de personas; este método ya se ha utilizado en otros estudios de etnia mapuche realizados en Chile..$^{23}$ En cuanto al uso de los apellidos para la identificación de etnia, puede afirmarse que se relacionan con factores genéticos (consignan la presencia de sus ancestros), no tanto con aspectos culturales ya que en la actualidad los indígenas chilenos escasamente practican ritos propios de su cultura, o hablan su lengua (mapudungun); desafortunadamente, en las bases de datos nacionales no se puede saber en qué medida están identificados los indígenas con su cultura. Si bien existen familias indígenas que han cambiado sus apellidos por los de origen chileno-español, éstas son la minoría. En fecha reciente, en los más de 7000 apellidos mapuches identificados, se ha logrado reconocer el cambio de apellidos 
en 3.6\% de ellos, por lo que el uso de los apellidos en los análisis de este artículo parece un buen método.

Entre las fortalezas se puede mencionar la inclusión en el análisis de más de 1500000 escolares de nivel primero básico, que representan casi el universo total de los que ingresan a la enseñanza pública chilena, en un país que realiza de forma sistemática mediciones antropométricas cada año en las escuelas y donde la casi totalidad de los escolares se matricula en establecimientos de enseñanza básica.

\section{Agradecimientos}

Agradecemos a la Junta Nacional de Auxilio Escolar y Becas del Ministerio de Educación, que facilitaron las bases de datos nacionales con la información de los escolares de primer año básico expuesta en este artículo.

\section{Referencias}

I. Pan American Health Organization. Situation of severe growth retardation in first-grade school children in countries of Central America around 2000. Epidemiol Bull 2004;25(I):9-13.

2. Rivera J, Monterrubio E, González-Cossío T, García-Feregrino R, García-Guerra A, Sepúlveda-Amor J. Estado nutricio de los niños indígenas menores de 5 años de edad en México: resultados de una encuesta nacional probabilística. Salud Publica Mex 2003;45(Supl 4): I I I

3. Chávez MC, Madrigal H,Villa A, Guarneros N. Alta prevalencia de desnutrición en la población infantil indígena mexicana. Encuesta Nacional de Nutrición 1999. Rev Esp Salud Publica 2003;77:245-255.

4. Psacharopoulos $\mathrm{G}$, Patrinos $\mathrm{H}$. Indigenous people and poverty in Latin America: an empirical analysis. World Bank Regional and Sectoral Studies. Washington, DC:World Bank, 1994.

5. Caballero E, Himes JH, Lohman T, Davis S, Stevens J, Evans M, et al. Body composition and overweight prevalence in 1704 schoolchildren from 7 American Indian communities. Am J Clin Nutr 2003;78:308-3/2. 6. Bernard L, Lavallée C, Gray-Donald K, Delisle H. Overweight in Cree schoolchildren and adolescents associated with diet, low physical activity, and high television viewing. J Am Diet Assoc 1995;95:800-802

7. Young TK, Dean HJ, Flett B, Wood-Steiman P. Childhood obesity in a population at high risk for type 2 diabetes. J Pediatr 2000; 136:365-369.
8. Hanley A, Harris S, Gittelsohn J,Wolever T, Saksvig B, Zinman B. Overweight among children and adolescents in a Native Canadian community: prevalence and associated factors. Am J Clin Nutr 2000;7I:693-700.

9. Beaton GH, Bengoa JM. Practical population indicators of health and nutrition. In: Beaton, GH, Bengoa, JM, Ed. Nutrition in Preventive Medicine. Geneva:WHO, 1976:590. Monograph series № 62.

10. Ministerio de Educación de Chile. Manual de salud escolar... Una tarea de todos. Edición 2006. [Consultado el 10 de septiembre de 2008]. Disponible en: http://www.junaeb.cl/manual_salud/JUNAEB.pdf. II. CDC/NCHS: CDC growth charts: United States 2000. [Consultado el I0 de septiembre de 2007]. Disponible en: http://www.cdc.gov/ growthcharts.

12. Ministerio de Planificación, Chile. Ficha CAS: un instrumento de focalización de programas sociales. [Consultado el 30 de marzo de 2007]. Disponible en: http:// www.mideplan.cl.

13. Larrea C, Freire W. Social inequality and child malnutrition in four Andean countries. Rev Panam Salud Publica 2002; I :356-364. 14. Larrea C, Karachi I. Does economic inequality affect child malnutrition? The case of Ecuador. Soc Sci Med 2005;60: 165-178. 15. Bustos P,Amigo H, Muñoz S, Martorell R. Growth in indigenous and non indigenous Chilean schoolchildren from 3 poverty strata. Am J Public Health 2001;91:1645-1649.

16. Bustos P,Weitzman M,Amigo H. Crecimiento en talla de niños indígenas y no indígenas chilenos. Arch Latinoam Nutr 2004:54:190-195. 17. Martorell R, Haschke F. Nutrition and growth. Nestle Nutrition Workshop Series, Pediatric Program. Philadelphia (PA): Lippincott Williams and Wilkins, 200I.

18. Ministerio de Salud de Chile. Encuesta Nacional de Salud 2003.

[Consultado el 20 de agosto de 2007]. Disponible en: http://epi.minsal.cl/ epi/html/invest/ENS/informeFinalENS.pdf.

19. Adjemián D, Bustos P,Amigo H. Nivel socioeconómico y estado nutricional: un estudio en escolares. Arch Latinoam Nutr 2007;57:125-129. 20. García V, Amigo H, Bustos P. Ingesta alimentaria en escolares chilenos de procedencia indígena y no indígena de diferente vulnerabilidad social. Arch Latinoam Nutr 2002;52:368-373.

21. Kain J, Uauy R, Lera L, Taibo M, Albala C. Trends in height and BMI of 6 -year-old children during the nutrition transition in Chile. Obes Res 2005; |3:2178-2।86.

22. Amigo H, Erazo M, Bustos P,Aguilar C, Taibo M.Vigilancia nutricional en escolares chilenos: validez de la información. Rev Med Chile 2008; 136:989-995.

23. Rojas F. Poverty determinants of acute respiratory infections among Mapuche indigenous peoples in Chile's Ninth Region of Araucania, using GIS and spatial statistics to identify health disparities. Int J Health Geogr 2007;6:26. 